\title{
ANÁLISE DA RELAÇÃo DE ASPECTOS CLIMÁTICOS NA GESTÃo DE ÁGUA DO AGRESTE PERNAMBUCANO
}

\author{
Thiago Henrique de Sales Cavalcante (UFPE) thiago.salescavalcante@ufpe.br \\ Layza Sayara Sobral Melo (UFPE) layza9623@gmail.com \\ Marcele Elisa Fontana (UFPE) marcele.elisa@ufpe.br
}

\section{Resumo}

A região do Agreste pernambucano caracteriza-se por uma área bastante sujeita a secas, devido às incertezas associadas ao clima semiárido e seco da região. Isso resulta no aumento da complexidade do gerenciamento do seu balanço hídrico. Assim, o presente artigo teve como objetivo estudar a influência de variáveis climáticas sobre a distribuição de água na região agreste de Pernambuco. Para isso, inicialmente foi realizado uma revisão sistemática da literatura para identificar quais são as variáveis mais relevantes na gestão de recursos hídricos em locais com clima semelhante ao do agreste pernambucano. Além disso, uma entrevista não estruturada foi realizada com especialista na área no agreste. Como principal resultado notouse que a utilização de variáveis relativas a atividades humanas, como estatísticas de industrialização, agricultura e pecuária, agrega no processo de gestão. Essas apontam que, o impacto das atividades humanas é mais relevante que os próprios aspectos climáticos na gestão de recursos-hídricos, mesmo em regiões com domínios climáticos desfavoráveis, como semiárido e árido.

\section{Palavras-Chaves: Gestão Hídrica; Seca; Agreste pernambucano; aspectos climáticos.}

\section{Introdução}

Muitas regiões do mundo estão passando por algumas das piores condições de seca por várias gerações. Em 2014, o Brasil experimentou sua pior seca em 80 anos, enquanto a Califórnia enfrentou recentemente sua pior seca por mais de um século. Durante a última década, secas prolongadas e severas também foram experimentadas na África, no sul da Europa e na Austrália, que passa pela maior seca do século, com prejuízos socioeconômicos, além de incêndios florestais com morte de mais de 1 bilhão de animais (PERUCHI, 2018). Projeções sugerem que as secas serão mais frequentes e severas em muitas partes do mundo, como resultado do crescimento populacional, do aumento da renda e das mudanças nas condições climáticas (IPCC, 2014).

Assim, é crescente a correlação dessas secas com as alterações climáticas, que geram impacto no padrão de precipitação, frequência e distribuição de inundações e secas e aumentam a taxa 
de evapotranspiração em diferentes regiões do mundo (FREDERICK \& MAJOR, 1997; FICKLIN et al., 2012).

Neste sentido, o nordeste brasileiro é marcado por instabilidades climatológicas, variando as condições de acordo com a localidade. A região do Agreste, por exemplo, é uma área bastante sujeita à secas. Por outro lado, a região é muito sujeita a inundações, ocasionadas pela falta de estrutura para suportar um alto volume de precipitações em um período muito curto (CUNHA et al., 2012).

Frente a esse cenário climático, a gestão de água local tradicionalmente submete todas as decisões estratégicas aos níveis dos reservatórios de água da região, o que implica em uma gestão de racionamento que se sustenta por todo o período de escassez de chuvas. O sistema de gestão se demonstrou ineficiente, visto que, segundo dados encontrados no site da Agencia Pernambucana de Águas e Clima (APAC, 2020), o maior reservatório da região está indisponível para abastecimento desde 2016, devido ao baixo nível de capacidade.

Contraditoriamente, a região do Agreste pernambucano possui sua economia movida por atividades que operam com elevados volumes de água no seu processo produtivo, tais como fabricação e lavagem de peças de jeans, criação bovina para corte e agropecuária. Além de ser a segunda maior bacia leiteira do Nordeste (produção diária de 2 milhões de litros). (CAVALCANTE, 2018; DIARIO DE PERNAMBUCO, 2020).

Em decorrência destas variações da oferta, a população da região reage diferentemente às políticas de racionamento impostas, o que resulta em diversos impactos para a população. Musolino et al. (2017), identificaram que diferentes grupos sociais regionais experimentaram diferentes consequências provenientes de uma mesma política de racionamento de água. Cavalcante et. al.; (2019), comprovaram que em determinada amostra da região é possível perceber essas diferentes reações à política de racionamento, bem como a mudança de hábitos regionais como resposta a instabilidade climática. Por isso, é fundamental o investimento em projetos estratégicos de forma direcionada.

Um indício de que a gestão de água não vem sendo eficiente, é o fato de 61 dos 71 municípios que compões o Agreste pernambucano entraram em estado de emergência em janeiro de 2020 por causa da seca (DIARIO DE PERNAMBUCO, 2020).

Uma das medidas iniciais a ser adotada pelas empresas abastecedoras para uma gestão eficiente a longo prazo, que inclua todo o período do ciclo da seca, é estudar quais as variáveis que possuem forte influência na tomada de decisão (FELFELANI \& KERACHIAN, 2016; CAVALCANTE, 2018). Desta forma, é fundamental a qualificação, dentre uma gama de 
fatores influenciadores, dos mais relevantes para tomada de decisões da gestão (FONTANA \& MORAIS, 2016), já que ações na área de recursos hídricos trazem consideráveis impactos em todos os segmentos da sociedade. Entretanto, em regiões que sofrem com a seca, o estudo dessas variáveis é uma ação complexa. A demanda da região, além de influência climática, sofre restrições de oferta, manejo político, perda de volume na distribuição, e etc.

Assim, dado que os problemas de abastecimento de água decorrentes da má gestão dos recursos hídricos frente os fatores climáticos envolvem a sociedade como um todo (FONTANA \& MORAIS, 2015), o presente artigo teve como objetivo estudar a influência de variáveis climáticas sobre a distribuição de água na região do agreste de Pernambuco. Para isso foi realizada uma revisão sistemática da literatura para identificação de aspectos climáticos que são relevantes para o gerenciamento de água em regiões com propriedades climáticas semelhantes às da região Agreste Pernambucana. Além disso, uma entrevista não estruturada com um especialista na área foi realizada para ampliar a compreensão do problema e vincular as estratégias de gestão estudadas na literatura com a realidade da região do agreste de Pernambuco.

Dessa forma, essa análise pode auxiliar empresas abastecedoras de água e gestores públicos no alinhamento do planejamento e gestão de água em períodos de estiagem com tendências utilizadas em regiões com características climáticas semelhantes.

Além desta introdução, o trabalho foi dividido em outras 4 secções a saber: Na seção 2, foram expostos os conceitos que fundamentam a realização dessa pesquisa. A seção 3 fornece informações sobre a metodologia adotada para esta pesquisa. Na seção 4, os resultados foram expostos e discutidos. A seção 5 foi dedicada à conclusão do trabalho.

\section{Fundamentação teórica}

A água é um recurso de uso público e de fundamental importância na vida de todos. Mesmo sendo um recurso tido pelo senso comum como abundante no mundo, a água em condições de uso pela população é, na verdade, escassa e a depender da região o acesso a esse recurso pode ser precário. Isso porque, o aumento dos níveis das fontes de água utilizadas para abastecimento público depende estritamente da precipitação das chuvas, que ocorrem de maneira desigual geográfica e periodicamente (CAMBRAINHA, 2015).

Em muitas regiões semiáridas do mundo, a escassez de recursos hídricos, o aumento da população e a experiência de secas estão exercendo grandes pressões sobre os recursos hídricos. Prevê-se que as mudanças climáticas exacerbem a vulnerabilidade dos recursos hídricos, o que 
destaca claramente a necessidade de desenvolver políticas sustentáveis de gestão da água e estratégias de adaptação apropriadas (HERRERA-PANTOJA \& HISCOCK, 2015).

Para se preparar para os desafios futuros, os tomadores de decisão precisam de metodologias que levem em conta cenários de mudanças climáticas em nível regional para prever efeitos prováveis nos recursos hídricos. A avaliação dos prováveis impactos das mudanças climáticas nos recursos hídricos é de grande importância para entender como diferentes setores podem ser afetados; e, além disso, desenvolver políticas sustentáveis de gestão da água e estratégias de adaptação apropriadas que permitam administrar com êxito a variabilidade da demanda de água, conflitos sobre a alocação de recursos hídricos e produzir esquemas de emergência em situações de seca e inundação (NKHONJERA \& DINKA, 2017; HERRERA-PANTOJA \& HISCOCK, 2015).

Além disso, tradicionalmente, políticas de racionamento de água são utilizadas nesse cenário de seca. Porém, essas políticas podem causar perdas socioeconômicas consideráveis, tais como restrição da produção industrial e agropecuária (HOWITT et al., 2015; HLALELE et al., 2016).

\section{Metodologia}

Trata-se de um estudo descritivo, de revisão sistemática de literatura (RSL), de natureza qualitativa, que teve como fonte de investigação artigos publicados no período de janeiro de 2015 à fevereiro de 2020.

A RSL é uma metodologia que busca localizar estudos existentes, para avaliar as suas contribuições, podendo assim saber o que é e o que não é conhecido sobre um determinado tema (DENYER \& TRANFIELD, 2009). Esse tipo de busca disponibiliza um resumo das evidências relacionadas a uma estratégia de intervenção específica, mediante a aplicação de métodos explícitos e sistematizados de busca, apreciação crítica e síntese da informação selecionada. Os parâmetros da RSL são expostos no Quadro 1. 


\begin{tabular}{|l|l|}
\hline & $\begin{array}{l}\text { Rq1: Quais são as principais variáveis usadas para } \\
\text { Perguntas de pesquisa }\end{array}$ \\
& $\begin{array}{l}\text { Rq2: Quais os principais resultados do uso dessas } \\
\text { variáveis na tomada de decisão de distribuição de } \\
\text { água? }\end{array}$ \\
\hline Palavras chaves & "climatic variables" "water management" "semiarid" \\
\hline Operador booleano & AND \\
\hline Intervalo de tempo & $2015-2020$ \\
\hline Bases de dados & Scopus + Science Direct \\
\hline
\end{tabular}

Fonte: Os autores (2020)

Posteriormente, foi realizada a leitura sistematizada dos estudos que passaram pela triagem e tiveram seus conteúdos explorados para uma melhor compreensão das diferentes perspectivas sobre os problemas abordados e das estratégias utilizadas dentro das publicações levantadas. Os estudos que não contemplaram os critérios de elegibilidade foram excluídos com base em critérios de relevância para a pesquisa.

Em seguida, foi realizada uma entrevista não estruturada com um especialista com mais de 30 anos de experiência na área. A entrevista abordou questões acerca da influência de aspectos climáticos na tomada de decisão da empresa, bem como monitoramento de dados e planejamento estratégico da empresa em relação a projetos atuais e futuros. Os resultados obtidos foram expostos e discutidos na seção seguinte.

\section{Resultados e discussões}

Após a primeira busca ser realizada, foram encontrados 343 artigos. O primeiro critério de seleção foi o intervalo de tempo, após selecionar apenas os artigos dos últimos 6 anos, restaram 178 artigos, distribuídos pelos anos como mostra na Figura 1.

Figura 1 - Publicações/ano

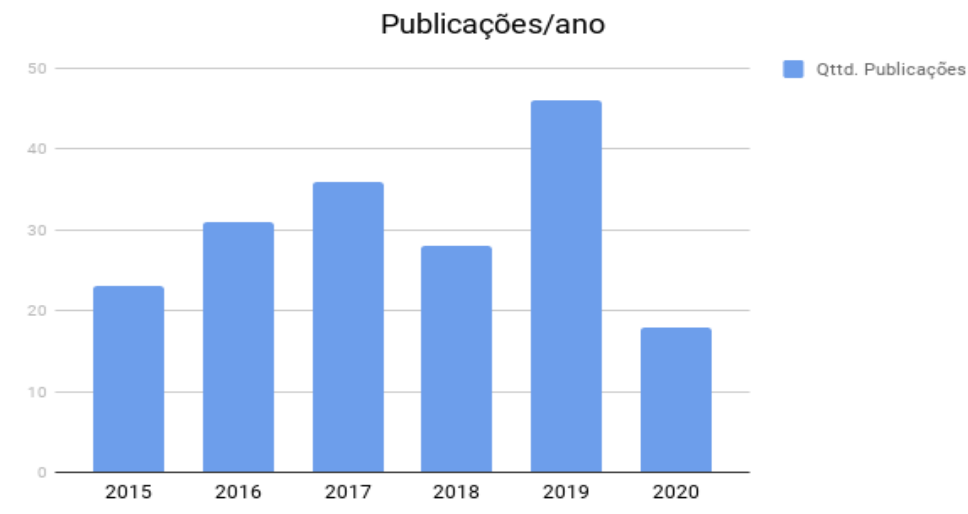

Fonte: Os autores (2020) 
Ao serem retirados os capítulos de livros, enciclopédias e resumos de congresso, restaram 159 artigos de pesquisa e de revisão. Analisando os títulos, foram excluídos artigos que não tratavam do tema de pesquisa, restando 50 artigos. Depois disso, analisou-se o clima a que se tratava os estudos, e 10 artigos foram selecionados por apresentarem uma localidade com clima similar ao agreste pernambucano. Estes trabalhos foram sumarizados no Quadro 2.

Quadro 2 - Resultados sumarizados

\begin{tabular}{|c|c|c|c|c|}
\hline Autor(es) & $\begin{array}{l}\text { Local de } \\
\text { estudo }\end{array}$ & $\begin{array}{c}\text { Clima } \\
\text { local }\end{array}$ & Variáveis & Principais resultados \\
\hline $\begin{array}{c}\text { ADHAM et } \\
\text { al., } 2019\end{array}$ & Tunísia & $\begin{array}{c}\text { Semiárid } \\
\text { o }\end{array}$ & $\begin{array}{l}\text { - Dados diários de vazão dos } \\
\text { reservatórios; } \\
\text { - Dados diários de influxo dos } \\
\text { reservatórios; } \\
\text { - Dados meteorológicos de } \\
\text { diferentes estações } \\
\text { (precipitação, temperatura, } \\
\text { umidade relativa e etc.); }\end{array}$ & $\begin{array}{l}\text { - Alterando as direções do fluxo } \\
\text { combinadas com o aumento da altura } \\
\text { dos vertedouros teve um grande } \\
\text { impacto no desempenho das } \\
\text { estruturas de captação de água da } \\
\text { chuva. } \\
\text { - O gerenciamento da água e o } \\
\text { design da estrutura desempenham } \\
\text { um papel mais importante no } \\
\text { desempenho da distribuição de água } \\
\text { da chuva do que na própria mudança } \\
\text { climática. }\end{array}$ \\
\hline $\begin{array}{c}\text { AHN \& } \\
\text { MERWAD } \\
\text { E, } 2014\end{array}$ & $\begin{array}{l}\text { Indiana, } \\
\text { Nova } \\
\text { York, } \\
\text { Arizona } \\
\text { e área da } \\
\text { Geórgia }\end{array}$ & $\begin{array}{l}\text { Continen } \\
\text { tais secas } \\
\text { e } \\
\text { subtropic } \\
\text { al seca }\end{array}$ & $\begin{array}{l}\text { - Dados meteorológicos } \\
\text { (precipitação, evapotranspiração } \\
\text { potencial e evaporação) } \\
\text { - Dados de vazão dos } \\
\text { reservatórios (vazão mensal e } \\
\text { influxos) }\end{array}$ & $\begin{array}{l}\text { - As atividades humanas podem } \\
\text { impactar a vazão de várias maneiras } \\
\text { através da urbanização, } \\
\text { desenvolvimento agrícola, e etc. } \\
\text { - O impacto humano é } \\
\text { significativamente maior em } \\
\text { comparação ao impacto climático. }\end{array}$ \\
\hline $\begin{array}{l}\text { CHANGBI } \\
\text { N et al., } \\
2019\end{array}$ & China & $\begin{array}{l}\text { Clima } \\
\text { árido e } \\
\text { semiárid } \\
\text { o }\end{array}$ & $\begin{array}{l}\text { - Dados meteorológicos de } \\
\text { diferentes estações } \\
\text { (precipitação, temperatura } \\
\text { média, e etc.); } \\
\text { - Dados mensais de } \\
\text { evapotranspiração; } \\
\text { - Dados de atividades humanas } \\
\text { ligadas ao reservatório. }\end{array}$ & $\begin{array}{l}\text { - A eficácia das mudanças climáticas } \\
\text { e as regulamentações humanas sobre } \\
\text { a variação de utilização de água } \\
\text { podem compensar umas às outras em } \\
\text { larga escala ou durante um longo } \\
\text { período de tempo; }\end{array}$ \\
\hline $\begin{array}{l}\text { GUPTA \& } \\
\text { JAIN, } 2017\end{array}$ & Índia & $\begin{array}{c}\text { Semiárid } \\
\text { o }\end{array}$ & $\begin{array}{l}\text { - Dados meteorológicos de } \\
\text { diferentes estações } \\
\text { (precipitação, temperatura, } \\
\text { umidade relativa e etc.); }\end{array}$ & $\begin{array}{l}\text {-O desenvolvimento do cenário dos } \\
\text { recursos reflete o declínio na } \\
\text { disponibilidade de reservas de água e } \\
\text { o aumento da demanda. } \\
\text {-Para tornar o sistema sustentável, é } \\
\text { necessário adotar métodos eficientes } \\
\text { de uso de água, bem como manejo de } \\
\text { águas de reuso. }\end{array}$ \\
\hline $\begin{array}{c}\text { HERRERA- } \\
\text { PANTOJA } \\
\& \\
\text { HISCOCK, } \\
2015\end{array}$ & México & $\begin{array}{c}\text { Semiárid } \\
\mathrm{o}\end{array}$ & $\begin{array}{l}\text { - Dados diários de vazão dos } \\
\text { reservatórios; } \\
\text { - Dados diários de influxo dos } \\
\text { reservatórios; } \\
\text {-Dados meteorológicos } \\
\text { (temperatura mínima, } \\
\text { temperatura máxima, } \\
\text { evaporação e precipitação). }\end{array}$ & $\begin{array}{l}\text { - Os cenários das mudanças } \\
\text { climáticas descrevem que, para o } \\
\text { futuro, os períodos seco e chuvoso se } \\
\text { tornarão mais frequentes. } \\
\text { - Indica que os gerentes de água } \\
\text { precisam revisar as políticas e } \\
\text { regulamentos atuais com uma } \\
\text { abordagem holística. }\end{array}$ \\
\hline
\end{tabular}




\begin{tabular}{|c|c|c|c|c|}
\hline $\begin{array}{c}\text { NKHONJE } \\
\text { RA \& } \\
\text { DINKA, } \\
2017\end{array}$ & África & $\begin{array}{l}\text { Semiárid } \\
\mathrm{o}\end{array}$ & $\begin{array}{l}\text { - Dados meteorológicos de } \\
\text { diferentes estações } \\
\text { (precipitação, temperatura, } \\
\text { umidade relativa e etc.); } \\
\text { - Dados de atividades humanas } \\
\text { ligadas ao reservatório. }\end{array}$ & $\begin{array}{l}\text {-Aspectos climáticos relacionados à } \\
\text { água, têm sérias implicações para o } \\
\text { desenvolvimento dos países. } \\
\text {-Nota-se novamente que a } \\
\text { incapacidade de abordar as medidas } \\
\text { de adaptação e mitigação dos } \\
\text { impactos das mudanças climáticas. }\end{array}$ \\
\hline $\begin{array}{l}\text { SHARMA, } \\
\text { et al., } 2019\end{array}$ & Índia & $\begin{array}{l}\text { Predomi } \\
\text { nante } \\
\text { Semiárid } \\
\text { o }\end{array}$ & $\begin{array}{l}\text { - Dados diários de vazão dos } \\
\text { reservatórios; } \\
\text { - Dados diários de influxo dos } \\
\text { reservatórios; } \\
\text { - Dados populacionais da última } \\
\text { década; } \\
\text { - Estatísticas de produção de } \\
\text { safras sazonais. }\end{array}$ & $\begin{array}{l}\text { - Atividades antropogênicas } \\
\text { contribuem com uma média de } 85 \% \\
\text { na queda de disponibilidade de água } \\
\text { superficial. Enquanto a variabilidade } \\
\text { da precipitação contribui com uma } \\
\text { média de } 15 \% \text {. } \\
\text { - O aumento da população e a } \\
\text { diminuição da disponibilidade de } \\
\text { água podem agravar as questões de } \\
\text { escassez de água. }\end{array}$ \\
\hline $\begin{array}{c}\text { SHEN et al., } \\
2017\end{array}$ & China & $\begin{array}{l}\text { Tempera } \\
\text { do e } \\
\text { árido }\end{array}$ & $\begin{array}{l}\text { - Dados meteorológicos de } \\
\text { diferentes estações } \\
\text { (precipitação, temperatura } \\
\text { média, e etc.); } \\
\text { - Dados das barragens e } \\
\text { reservatórios (profundidade da } \\
\text { lâmina d'água e vazão); } \\
\text { - Dados de cobertura vegetal nas } \\
\text { proximidades do reservatório. }\end{array}$ & $\begin{array}{l}\text { - A área de estudo apresentou relação } \\
\text { significativamente positiva entre o } \\
\text { índice de vegetação das proximidades } \\
\text { dos reservatórios e a precipitação } \\
\text { local; } \\
\text { - As atividades antropológicas } \\
\text { realizadas na área das proximidades } \\
\text { dos reservatórios interferem no } \\
\text { processo de evaporação da água dos } \\
\text { reservatórios. }\end{array}$ \\
\hline $\begin{array}{c}\text { SINISCAL } \\
\text { CHI et al., } \\
2018\end{array}$ & $\begin{array}{c}\text { Argentin } \\
\mathrm{a}\end{array}$ & $\begin{array}{l}\text { Predomi } \\
\text { nante } \\
\text { Semiárid } \\
\text { o }\end{array}$ & $\begin{array}{lr}\text {-Dados } & \text { meteorológicos } \\
\text { (temperatura média, radiação, } \\
\text { exposição ao sol, evaporação } \\
\text { potencial, umidade relativa, } \\
\text { velocidade dos ventos e } \\
\text { precipitação) }\end{array}$ & $\begin{array}{l}\text {-O amortecimento das oscilações } \\
\text { hidrológicas, preservação dos fluxos } \\
\text { ambientais e conectividade entre os } \\
\text { corpos d'água aumentam a eficiência } \\
\text { do sistema de abastecimento. }\end{array}$ \\
\hline $\begin{array}{c}\text { XING et al., } \\
2018\end{array}$ & China & $\begin{array}{l}\text { Clima } \\
\text { árido e } \\
\text { semiárid } \\
\text { o }\end{array}$ & $\begin{array}{l}\text { - Dados mensais de } \\
\text { precipitação; } \\
\text { - Dados mensais de } \\
\text { evapotranspiração; } \\
\text { - Estatísticas mensais do grau de } \\
\text { aridez; } \\
\text {-Dados mensais de escoamento. }\end{array}$ & $\begin{array}{l}\text {-A proposição e aplicação do modelo } \\
\text { Budyko-E fornecem uma nova } \\
\text { maneira de avaliar mensalmente os } \\
\text { recursos ecológicos e hídricos na } \\
\text { escala regional. }\end{array}$ \\
\hline
\end{tabular}

Fonte: Os autores (2020)

A partir dos resultados obtidos na revisão da literatura, observou-se que as restrições na oferta de água tornam mais difícil a manutenção do abastecimento de água para a população em geral. Além disso, há uma relação de proporcionalidade na variação da economia local com as consequências da falta de abastecimento nessas localidades (ex.: perda de produção, restrição de produção, perda de lavouras e etc.).

Entretanto, é relevante citar que, em muitas localidades, os aspectos climáticos não representam a maior influência nas decisões tomadas pelos gestores de água. As atividades econômicas desempenhadas na região se demonstram mais relevantes no processo de tomada de decisão. 
Adicionalmente, percebeu-se que a captação de dados precisos de reservatórios (vazão, influxo e etc.), bem como dados meteorológicos (perspectiva de precipitação, evapotranspiração, temperatura e etc.), permite um sistema de gerenciamento de água mais eficiente, por permitir a identificação prévia de grandes variações nos níveis de chuva ou de água, permitem planejamento de contingenciamento. Muitos desses dados são monitorados pela concessionária de água, porém, em uma frequência não suficiente para embasar a aplicação de projetos com a precisão necessária para o gerenciamento local.

Ao se tratar da região Agreste Pernambucano, o único meio de captação de água da região é o volume de água armazenado em reservatórios, pois a região não possui rios, e é pobre de lençóis freáticos. Porém, o fator da seca já é conhecido e monitorado, inclusive pela APAC, o que indica que deveria haver projetos de previsão e controle no longo prazo. Atualmente, apenas é notada a existência de projetos de contenção emergenciais, no qual é ofertado um apoio financeiro do governo ao município para aplicação de recursos (carros pipa, material, e etc.) nas atividades básicas da região mais afetada pela seca.

Estudos anteriores demonstram que, além das características climáticas, têm-se diversos fatores que dificultam a gestão de recursos hídricos na região. Dentre eles, as características diferentes de hábitos de consumo de água da região, bem como hábitos de consumo que só são presentes em período de restrição de água (CAVALCANTE et al., 2019). A entrevista junto ao especialista confirma isso, já que foi identificada uma variação do consumo de água por parte da população em períodos de racionamento e em períodos de oferta mais elevada de água.

Aditivamente, há registros de altos níveis de perda de água na distribuição, com níveis superiores a 50\% de água, sendo Pernambuco o estado com pior disponibilidade hídrica do país (FONTES, 2018). Esse volume de água, além de representar uma enorme perda de valor para a empresa abastecedora da região, poderia ser realocado e utilizado em cenários de restrição de oferta de chuva. Evitando, assim, racionamento extremo de água e situações críticas como a atual, que classificam mais de $85 \%$ dos municípios do agreste do estado em estado de emergência. Segundo o especialista consultado, há investimento na região para o controle de perdas, porém não o necessário para contingenciar esse volume perdido de água, por não se tratar de uma prioridade para a empresa. A falta de manutenção em toda a rede de distribuição é identificada como o fator de maior relevância para essa perda de volume.

Adicionalmente, o especialista identificou uma perspectiva grande para o gerenciamento de água da região com a construção de novas estruturas de abastecimento, como a Adutora do Agreste e a Transposição do rio São Francisco. Porém, erros de engenharia ocorridos 
anteriormente em obras da companhia foram relatados, inclusive inviabilizando projetos importantes de reaproveitamento de água.

\section{Conclusão}

Esse trabalho estudou a influência de aspectos climáticos sobre a gestão de água na região Agreste, considerando as especificidades da região. Foi constatada a importância de considerar no planejamento de utilização de recursos hídricos, não só os aspectos climáticos, mas principalmente as variáveis correspondentes às atividades humanas abastecidas pela rede de distribuição de água, pois essas têm mais impactos na distribuição de água. Em regiões como o Agreste pernambucano, que apresenta uma seca recorrente, é importante saber quantificar o impacto de cada uma das variáveis isoladamente, para assim traçar planos estratégicos voltados para o controle dessa influência.

Desta forma, percebeu-se a necessidade de mudança na estratégia de gerenciamento dos recursos, iniciando esse processo com a implementação de métodos mais eficientes na captação de dados da região. Visto que, no sistema atual, a empresa abastecedora não possui a maior parte dos dados necessários para aplicação de modelos de apoio a tomada de decisão. Essas mudanças trazem benefícios ao planejamento da utilização do recurso hídrico pela empresa em curto, médio e longo prazo.

Como trabalhos futuros propõe-se estudos direcionados as principais atividades econômicas desenvolvidas na região (como fabricação de jeans).

\section{REFERÊNCIAS}

ADHAM, Ammar; WESSELING, Jan G.; ABED, Rasha; RIKSEN, Michel. OUESSAR, Mohamed; RITSEMA, Coen J. Assessing the impact of climate change on rainwater harvesting in the Oum Zessar watershed in Southeastern Tunisia, Agricultural Water Management, v. 221 p. 131-140, 2019.

AGÊNCIA PERNAMBUCANA DE ÁGUAS E CLIMA - APAC. Monitoramento dos reservatórios, 2018. Disponível em <http://200.238.109.99:8080/apacv5/cons_monitora_web/cons_monitora_web.php> Acesso em 10/02/2020.

AHN Kuk-Hyun \& MERWADE Venkatesh. Quantifying the relative impact of climate and human activities on streamflow, Journal of Hydrology, 2014, v. 515, p. 257-266.

CAMBRAINHA, Géssika \& FONTANA, Marcele Elisa. Análise da aplicação de investimentos em perdas de água no nordeste brasileiro. Revista Eletrônica em Gestão, Educação e Tecnologia Ambiental, 2015, v. 19, n. 2 p.983-994.

CAVALCANTE, Thiago Henrique de Sales; FONTANA, Marcele Elisa; URTIGA, Marcela Maia. Diagnostico dos desafios à previsão de demanda por água devido à ocorrência de fenômenos climáticos na região do agreste pernambucano. In: XXXVIII ENEGEP, 2018, Maceió (AL). XXXVIII ENEGEP, 2018. 
CAVALCANTE, Thiago Henrique de Sales; FONTANA, Marcele Elisa; URTIGA, Marcela Maia. Estudo do comportamento de consumo de água no agreste de Pernambuco. In: XXXIX Encontro Nacional de Engenharia de Produção, 2019, Santos (SP). XXXIX ENEGEP, 2019.

CHANGBIN, Li; LIUMING, Wang; WANG, Wanrui; JIAGUO, Qi; YANG, Linshan; YUAN, Zhang; WU, Lei; XIA, Cui; PENG, Wang. An analytical approach to separate climate and human contributions to basin streamflow variability, Journal of Hydrology v. 559,p. 30-42, 2018.

CUNHA, Tassio; LINHARES, Franklin; SANTOS, José; VIANNA, Pedro. Mapeamento e tipologia dos conflitos pela gestão e controle das águas no Estado da Paraíba. Boletim de Geografia, 2012, Maringá, v. 30, n. 2, p. 31-43.

DENYER, D., \& TRANFIELD, D. Producing a systematic review. In D. A. Buchanan \& A. Bryman (Eds.), The Sage handbook of organizational research methods, 2009, (p. 671-689).

DIARIO DE PERNAMBUCO. Pernambuco decreta situação de emergência em 61 cidades do Agreste. Diario de Pernambuco, 2020. Disponível em:

https://www.diariodepernambuco.com.br/noticia/vidaurbana/2020/01/pernambuco-decreta-situacao-deemergencia-em-61-cidades-do-agreste.html. Acesso em: 10/02/2020.

FELFELANI, Farshid \& KERACHIAN, Reza. Municipal water demand forecasting under peculiar fluctuations in population: A case study of Mashhad, a tourist city. Hydrol Sci J, 2016 v. 61, n. 8, p.1524-1534.

FICKLIN, Darren L., STEWART, Iris T., MAURER, Edwin P. Effects of climate change on stream temperature, dissolved oxygen, and sediment concentration in the Sierra Nevada in California. Water Resources Research, v. 37 (1), pp. 7-23. 2013.

FONTANA, Marcele Elisa, MORAIS, Danielle Costa. Segmentation model for water distribution networks based on the characteristics of consumer units. Prod., São Paulo, v. 25 (1), pp. 143-156, 2015.

FONTANA, Marcele Elisa; MORAIS, Danielle Costa. Decision model to control water losses in distribution networks. Prod., São Paulo, v. 26 (4), pp. 688-697, 2016.

FONTES, Bruno. Pernambuco tem pior disponibilidade hídrica do país e metade da água se perde antes de chegar a torneiras, diz TCE . G1, 2018. Disponível em:

$<$ https://g1.globo.com/pe/pernambuco/noticia/2018/12/19/pernambuco-tem-pior-disponibilidade-hidrica-do-paise-metade-da-agua-se-perde-antes-de-chegar-a-torneira-diz-tce.ghtml >. Acesso em: 15 de fevereiro 2020.

FREDERICK, Kenneth D., MAJOR, David C. Climatic change and the water resources. Clim.Change v. 37 (1), pp. 7-23, 1997.

GUPTA, Rajiv \& JAIN, Gaurav Kumar. Scenario planning for water resource management in semi arid zone, Physics and Chemistry of the Earth, Parts A/B/C, v.105, 2018, p. 290-299.

HERRERA-PANTOJA, M. \& HISCOCK, Kevin M. Projected impacts of climate change on water availability indicators in a semi-arid region of central Mexico, Environmental Science \& Policy, v. 54, 2015, p. 81-89.

HLALELE, B.; MOKHATLE, I.M.; MOTLOGELOA, R.T. Assessing economic impacts of agricultural drought: a case of Thaba Nchu, South Africa Journal Earth Science Climate Change, 2016,v.7 p. 327.

HOWITT, Richard; MACEWAN, Ducan; MEDELLÍN-AZUARA, Josué; LUND, Jay; SUMNER, Daniel. Economic Analysis of the 2015 Drought for California Agriculture. University of California, Davis, CA 2015, Hydrogeology Journal, p. 16.

INTERGOVERNMENTAL PANEL ON CLIMATE CHANGE (IPCC).World Meteorological Organization.Relatório. (2014). Disponível em< https://www.ipcc.ch/report/ar5/wg3/ > acesso em 15 de fevereiro de 2020.

MUSOLINO, Dario; DE CARLI, Alessandro; MASSARUTTO, Antonio. Evaluation of socio-economic impact of drought events: the case of Po river basin, European Countryside, 2017, v. 9(1), p. 163-176. 
NKHONJERA, German K. \& Megersa O. Dinka, Significance of direct and indirect impacts of climate change on groundwater resources in the Olifants River basin: A review, Global and Planetary Change,v.158, 2017, p. $72-82$.

PERUCHI, Marcio. Seca na Austrália é a pior em 100 anos. Comprerural, 2018. Disponível em: < https://www.comprerural.com/seca-na-australia-e-a-pior-em-100-anos/>. Acesso em: 15 de fevereiro 2020.

SHARMA Priyank J.; PATEL, Prem L.; JOTHIPRAKASH, Vinayakam. Impact of rainfall variability and anthropogenic activities on streamflow changes and water stress conditions across Tapi Basin in India, Science of The Total Environment, 2019, v. 687, p. 885-897.

SHEN, Qin; GUANGYAO, Gao; YIHE, Lü; SHUAI, Wang; XIAOHUI, Jiang; BOJIE, Fu. River flow is critical for vegetation dynamics: Lessons from multi-scale analysis in a hyper-arid endorheic basin, Science of The Total Environment, 2017, v. 603-604,p. 290-298.

SINISCALCHI, Amira; KOPPRIO, Germán; RANIOLO, Luis Ariel.; GOMEZ, Eduardo Alberto; DIAZ, M.S.; LARA, R.J. Mathematical modelling for ecohydrological management of an endangered endorheic salt lake in the semiarid Pampean region, Argentina, Journal of Hydrology; vol. 563 p. 778 - 789, 2018.

XING, Wanqiu; WEIGUANG, Wang; QUANXI, Shao; BIN, Yong; CATHERINE, Liu; XIAOZHOU, Feng; QING, Dong. Estimating monthly evapotranspiration by assimilating remotely sensed water storage data into the extended Budyko framework across different climatic regions, Journal of Hydrology, 2018 v. 567, p. 684-695. 